head injury. Sixteen patients died; Motality was $5.7 \%$.

7 of 16 cases $(43.8 \%)$ were over fifty years old.

The most frequent cause of head injury was automobile accident and the second was fall.

9 of 16 cases $(56.3 \%)$ was admitted in several hours and was treated quickly.

3 cases died in 24 hours and 11 cases $(68.7 \%)$ died within one week. This shows the limit of neurosurgical therapy for severe head injury.

Clinical diagnosis, Neurological, General findings and treatment on these cases were analysed.

Normal GOT activity in C.S.F. does not necessarily indicate good prognosis.

Temporobasal linear fracture shown in plain craniogram accompanies with poor prognosis.

Extensive cerebral contusion, brain edema and pressure cone are the commonest findings at autopsy.

In the cases in whom respirator was used, the granular layer of the cerebellum diminished markedly.

\title{
77. Fluorescein Retinal Serialography in Posttraumatic Encephalopathy
}

\author{
Tsuneo OHONo \\ Dept. of Neurosurgery, Kanto Rosai Hospital \\ Yoshinao FUKADo \\ Dept. of Ophthalmology, Kanto Rosai Hospital
}

Fluorescein fundus photography, first described by Novotny and Alvis, may be an interesting method for neurologists and neurosurgeous to observe the cerebro-vascular dynamics. David et al. in 1966 reported that the speed of retinal circulation could be grossly estimated by the rapid serial fluorescein fundus photography, and concluded that in the patients with the occlusion of the internal carotid artery a remarkable delay was observed, not only in the arrival of fluorescein dye, but in the sequence of vascular filling which is normally seen.

We have employed this fluorescein retinal serialography in the patients after head injury for observation of retinal circulatory dynamics, which might be a model of the cerebral circulation. We estimated the retinal circulation by the degree of vascular dye-filling, and by the interval from the arrival to the disappearance of the dye of retinal vessels.

In Fig. 1 (normal individual), the normal vascular filling is demonstrated. 


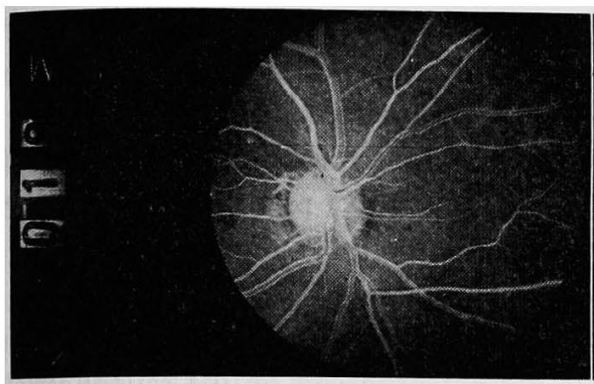

19 seconds after injection i.v.

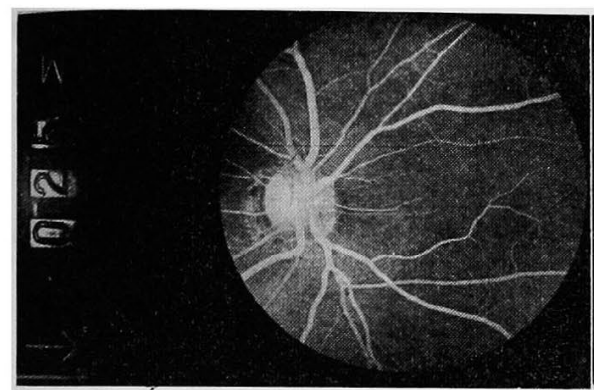

25 seconds after injection i.v.

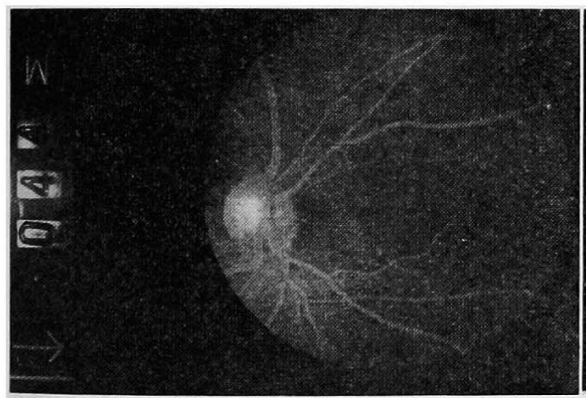

44 seconds after injection i.v.

Fig. 1. 41 year-old female. Normal filling and prologed fluorescence of the retinal vessels.

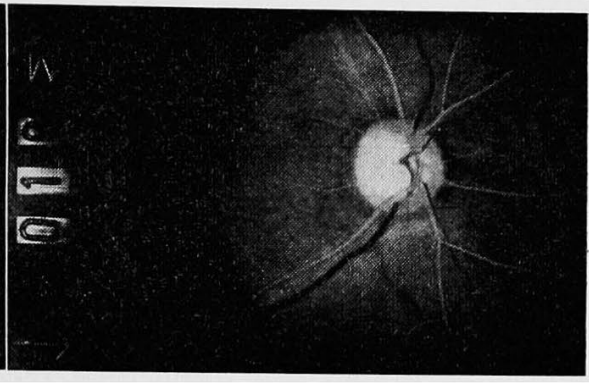

19 seconds after injection i.v.

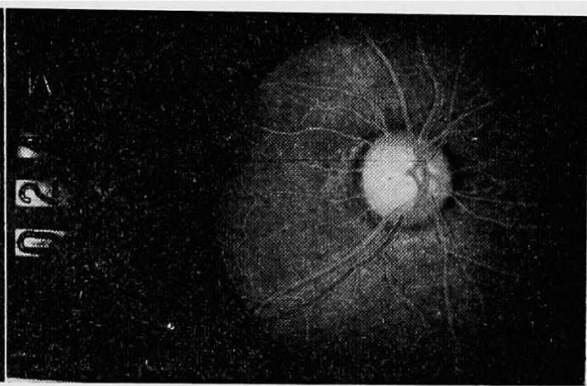

24 seconds after ihjection i.v.

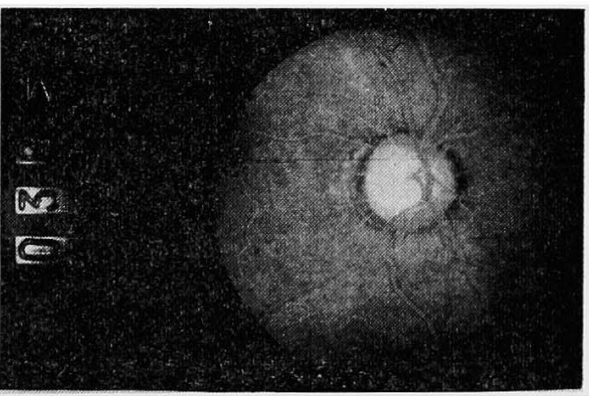

36 seconds after injection i.v.

Fig. 2. 42 year-old male with posttraumatic encephalopathy. Retarded insuffieicent filling and rapid disappearance of fluorescence of the retinal vessels.

In Fig. 2 (an abnormal case), the insufficient filling and the rapid disappearance (within 50 or 60 seconds) of the dye are shown.

Seventy five cases of posttraumatic encephalopathy aged 16 to 64 years (mean age 35.2) examined in this series (male 72 and female 3 ). In Table 1 the relationship between the findings of fluorescein retinal angiography, which were divided into abnormal and normal, and the clnical data are tabulated. 
(1) Of 46 patients with some neurological signs of central nervous system and/or cranial nerves, 23 cases (64\%) revealed abnormal findings of the retinal serialography,

(2) Of 44 cases with the abnormal retinal serialography, 33 patients (75\%) had some abnormalities of EEG. And 24 patients (55\%) revealed the remarkable EEG "build-up". On the contrary, in the normal retinal angiography group of 31 cases only 8 patients $(26 \%$ ) revealed the EEG "build-up".

(3) In cases of the commotio retinae, the orbital fracture and traumatic optic nerve atrophy, any distinct difference did not observed on the retinal serialography of the eye of the side of lesion as compared to the opposite eye.

(4) In conclusion, we suggest that the fluorescein retinal serialography might reveal not only the retinal circulation but the cerebrovascular dynamics or cerebral circulation.

Table 1.

\begin{tabular}{|c|c|c|c|c|c|}
\hline & & \multicolumn{4}{|c|}{ Fluorescein Retinal Angiography } \\
\hline & & \multicolumn{2}{|c|}{ Abnormal } & \multicolumn{2}{|r|}{ Abnormal } \\
\hline \multirow{4}{*}{ PTA } & Nil & 5 & & 4 & \\
\hline & $\leqq 1 \mathrm{hr}$ & 9 & & 11 & \\
\hline & $<24$ hrs. & 11 & & 4 & \\
\hline & $\geqq 24$ hrs. & 19 & & 12 & \\
\hline \multirow{2}{*}{\multicolumn{2}{|c|}{$\begin{array}{l}\text { Neurological } \\
\text { signs }\end{array}$}} & 21 & & 18 & \\
\hline & & 23 & atropny 3) & 13 & (optic n. atrophy 4) \\
\hline \multirow{4}{*}{ EEG } & Normal & 11 & & 18 & \\
\hline & Build-up & 24 & $25 \%$ & 8 & $26 \%$ \\
\hline & Marked LV & 7 & & 3 & \\
\hline & Asymmetry & 2 & & 2 & \\
\hline & Cases & 44 & & 31 & \\
\hline
\end{tabular}

\title{
78. Cerebrovascular Hemodynamics Following Head Trauma
}

\section{-With special emphasis on measurement of cerebrovascular calibers-}

\author{
Yaichi AkIBa, Kenshichiro Endo, Toshio Yamada and Ichiro OgaWA \\ 2nd Department of Surgery, Fukushima Medical College
}

It is a well- known fact that head trauma causes various kinds of intracranial 